\title{
The Be/X-ray transient 4U 0115+63/V635 Cassiopeiae
}

\section{Quasi-cyclic variability ${ }^{\star}$}

\author{
P. Reig ${ }^{1,2}$, V. Larionov ${ }^{3}$, I. Negueruela ${ }^{4}$, A. A. Arkharov ${ }^{5}$, and N. A. Kudryavtseva ${ }^{3,6}$
}

1 IESL, Foundation for Reseach and Technology-Hellas, 71110 Heraklion, Greece

2 Physics Department, University of Crete, 71003 Heraklion, Greece e-mail: pau@physics.uoc.gr

3 Astronomical Institute, St. Petersburg University, 198904 St. Petersburg, Russia e-mail: vml@vl1104.spb.edu

4 Departamento de Física, Ingeniería de Sistemas y Teoría de la Señal, Universidad de Alicante, 03080 Alicante, Spain e-mail: ignacio@dfists.ua.es

5 Pulkovo Central Astronomical Observatory, Pulkovskoe shosse 65, 196140 St. Petersburg, Russia e-mail: arkadi@arharov.ru

${ }^{6}$ Max-Planck-Institut fur Radioastronomie, Auf dem Huegel 69, 53121 Bonn, Germany

Received 9 August 2006 / Accepted 3 November 2006

ABSTRACT

Context. $4 \mathrm{U} 0115+63$ is one of the most active and best studied Be/X-ray transients. Previous studies of $4 \mathrm{U} 0115+63$ have led to the suggestion that $4 \mathrm{U} 0115+63$ undergoes relatively fast quasi-cyclic activity. However, due to the lack of good coverage of the observations, the variability time scales are uncertain.

Aims. Our objective is to investigate the long-term behaviour of $4 \mathrm{U} 0115+63 / \mathrm{V} 635$ Cas to confirm its quasi-cyclic nature and to explain its correlated optical/IR and X-ray variability.

Methods. We have performed optical/IR photometric observations and optical spectroscopic observations of 4U 0115+63/V635 Cas over the last decade with unprecedented coverage. We have focused on the $\mathrm{H} \alpha$ line variability and the long-term changes of the photometric magnitudes and colours and investigated these changes in correlation with the X-ray activity of the source.

Results. The optical and infrared emission is characterised by cyclic changes with a period of $\sim 5$ years. This long-term variability is attributed to the state of the circumstellar disc around the Be star companion. Each cycle involves a low state when the disc is very weak or absent and the associated low amplitude variability is orbitally modulated and a high state when a perturbed disc precesses, giving rise to fast and large amplitude photometric changes. X-ray outbursts in $4 \mathrm{U} 0115+63$ come in pairs, i.e., two in every cycle. However, sometimes the second outburst is missing.

Conclusions. Our results can be explained within the framework of the decretion disc model. The neutron star acts as the perturbing body, truncating and distorting the disc. The first outburst would occur before the disc is strongly perturbed. The second outburst leads to the dispersal of the disc and marks the end of the perturbed phase.

Key words. stars: pulsars: individual: 4U 0115+63 - stars: pulsars: individual: V635 Cas - X-rays: binaries - stars: neutron stars: binaries: close - stars: emission line, Be

\section{Introduction}

4U 0115+63 was one of the first Be/X-ray binaries to be discovered. The oldest available X-ray observation dates back to August 1969 when the Vela $5 B$ satellite detected the source as three small outbursts separated by 180 days (Whitlock et al. 1989). Since then about 15 outbursts have been reported (see Table 1). Normally, these outbursts represent an increase in the X-ray luminosity by a factor $\sim 100$ and last for about a month. The strongest outbursts reach luminosities close to the Eddington value $\left(\sim 10^{38} \mathrm{erg} \mathrm{cm}^{-2} \mathrm{~s}^{-1}\right)$. The lack of orbital modulation and the relative large increase in luminosity define these outbursts as type II. Only in one occasion, in 1996, has 4U 0115+63 showed orbitally modulated, shorter and smaller outbursts, i.e., type I (Negueruela et al. 1998). For a review of

* Tables 2 and 3 are only available in electronic form at the CDS via anonymous ftp to cdsarc.u-strasbg.fr (130.79.128.5) or via http://cdsweb.u-strasbg.fr/cgi-bin/qcat?]/A+A/462/1081 the X-ray variability in Be/X-ray binaries see, e.g., Coe (2000) and Ziolkowski (2002).

X-ray pulsations $\left(P_{\text {spin }}=3.6 \mathrm{~s}\right)$ were soon discovered (Cominsky et al. 1978) and the orbital parameters $\left(P_{\text {orb }}=\right.$ $24.3 \mathrm{~d}, e=0.34, a_{x} \sin i=140.1 \mathrm{lt}-\mathrm{s}$ ) determined (Rappaport et al. 1978). Subsequent detections led to the discovery of one (Wheaton et al. 1979), two (White et al. 1983), three (Heindl et al. 1999) and four (Santangelo et al. 1999) cyclotron resonance scattering features.

The 1978 outburst allowed the identification of the optical counterpart (Johns et al. 1978; Hutchings \& Crampton $1981)$ with a reddened $\left(A_{\mathrm{V}} \gtrsim 5\right.$ mag) B-type star that showed $\mathrm{H} \alpha$ in emission, V635 Cas. Extensive studies in the IR/optical (Kriss et al. 1983; Mendelson \& Mazeh 1991; Unger et al. 1998; Negueruela et al. 2001) and X-ray (Whitlock et al. 1989; Tsunemi \& Kitamoto 1988) bands allowed the determination of the astrophysical parameters - V635 Cas is a $V \approx 15 \mathrm{~B} 0.2 \mathrm{Ve}$ star located at a distance of $\sim 7-8 \mathrm{kpc}-$ and to the suggestion 
Table 1. Historical record of the X-ray outbursts and spin period of $4 \mathrm{U} 0115+63$.

\begin{tabular}{|c|c|c|c|c|c|c|}
\hline Date & Satellite & $\begin{array}{c}\text { Intensity } \\
\text { (Crab) }\end{array}$ & Reference & MJD & $P_{\text {spin }}$ & Reference \\
\hline 1969 Aug. & Vela 5B & 0.38 & Whitlock et al. (1989) & 40963 & $3.614658 \pm 0.000036$ & Kelley et al. (1981) \\
\hline 1970 Jan. & Vela 5B & 0.23 & Whitlock et al. (1989) & 42283 & $3.6142 \pm 0.0001$ & Whitlock et al. (1989) \\
\hline 1970 Aug. & Vela 5B & 0.22 & Whitlock et al. (1989) & 43556 & $3.61355 \pm 0.000000$ & Johns et al. (1978) \\
\hline 1971 Jan. & Uhuru & 0.17 & Forman et al. (1976) & 44589 & $3.6146643 \pm 0.0000018$ & Ricketts et al. (1981) \\
\hline 1974 Aug. & Vela 5B & 1.8 & Whitlock et al. (1989) & 47932 & $3.614690 \pm 0.000002$ & Tamura et al. (1992) \\
\hline \multirow[t]{3}{*}{1978 Jan. } & SAS 3 & 1.7 & Cominsky et al. (1978) & 47942 & $3.61461 \pm 0.00001$ & Lutovinov et al. (2000) \\
\hline & Ariel 5 & 0.5 & Rose et al. (1979) & 49481 & $3.6145107 \pm 0.0000010$ & Scott et al. (1994) \\
\hline & HEAO 1 & 0.96 & Johns et al. (1978) & 50042 & $3.614499 \pm 0.000004$ & Finger et al. (1995) \\
\hline 1980 Dec. & Ariel 6 & 0.18 & Ricketts et al. (1981) & 50307 & $3.614452 \pm 0.000003$ & Scott et al. (1996) \\
\hline 1987 Feb. & Ginga & 0.18 & Tsunemi \& Kitamoto (1988) & 51232 & $3.61452 \pm 0.00001$ & Wilson et al. (1999) \\
\hline \multirow[t]{2}{*}{1990 Feb. } & Ginga & 0.4 & Tamura et al. (1992) & 53261 & $3.614 \pm 0.003$ & Zurita-Heras et al. (2004) \\
\hline & GRANAT & 0.8 & Lutovinov et al. (2000) & 53266 & $3.616 \pm 0.001$ & Tueller (2004) \\
\hline 1991 Apr. & CGRO & 0.08 & Cominsky et al. (1994) & & & \\
\hline 1994 May & CGRO & 0.18 & Wilson et al. (1994) & & & \\
\hline \multirow[t]{2}{*}{1995 Nov. } & CGRO & 0.07 & Finger et al. (1995) & & & \\
\hline & GRANAT & 0.7 & Sazonov \& Sunyaev (1995) & & & \\
\hline \multirow[t]{3}{*}{1999 Mar. } & RXTE & 0.4 & This work & & & \\
\hline & BeppoSAX & 0.3 & Santangelo et al. (1999) & & & \\
\hline & CGRO & $>0.07$ & Wilson et al. (1999) & & & \\
\hline 2000 Sep. & RXTE & 0.2 & This work & & & \\
\hline \multirow[t]{2}{*}{2004 Sep. } & RXTE & 0.3 & This work & & & \\
\hline & INTEGRAL & 0.4 & Zurita-Heras et al. (2004) & & & \\
\hline
\end{tabular}

Vela 5B: 3-12 keV, Uhuru: 2-6 keV, HEAO 1: 3-13 keV, SAS 3: 1-27 keV, Ariel 5: 3-6 keV, Ariel 6: 1-50 keV,Ginga: 1-20 keV. CGRO: 20-50 keV, GRANAT: 8-20 keV, RXTE: 2-12 keV, BeppoSAX: 2-10 keV, INTEGRAL: 20-60 keV.

of cyclic changes with a quasi-period of 3-5 years. This quasicycling behaviour is closely related to the dynamical evolution of the viscous circumstellar disc around the Be star. The Be star loses and reforms the disc on time scales of 3-5 years (Negueruela et al. 2001). At some point during the growing phase the disc becomes unstable and highly disturbed.

In this paper we present the most complete monitoring of $4 \mathrm{U} 0115+63 / \mathrm{V} 635$ Cas in the optical and infrared wavelength bands made up to now. Our observations are used in combination with published data to investigate its quasi-cyclic optical/IR/Xray variability. The results presented here build on previous work by Negueruela \& Okazaki (2001, Paper I) and Negueruela et al. (2001, Paper II).

\section{Observations}

\subsection{Optical and infrared photometry}

Optical ( $B V R I)$ photometric observations were obtained from the $20 \mathrm{~cm}$ (before April 2001) and $70 \mathrm{~cm}$ (after April 2001) telescopes at the Crimean Observatory (CRI) in Ukraine between 1998-2005 using an ST-7 CCD of the St. Petersburg University. The array is $765 \times 510$ pixels, corresponding to a $8.1^{\prime} \times 5.4^{\prime}$ field of view $\left(0.65^{\prime \prime} /\right.$ pixel scale). The data set consists of about $260 \mathrm{~B}$ and 350 VRI measurements. In order to improve the signal-tonoise ratio, up to 5 images in each colour band were obtained and co-added to create the final image. The standard technique of bias and dark subtraction and flat-fielding was used. A calibrated set of standard stars, located within the same field, was used to perform aperture photometry using a SExtractor-based package. The photometric accuracy is usually better than 0.01 in $V, R$ and $I$ and about 0.03 in $B$.

Near-infrared photometric data (about $200 \mathrm{JHK}$ measurements) were obtained at Campo Imperatore (Italy) with SWIRCAM NIR camera with the PICNIC $256 \times 256$ pixel array, attached to the $1.1 \mathrm{~m}$ telescope. The camera's field of view is $4.5^{\prime} \times 4.5^{\prime}$ with a $1.04^{\prime \prime} /$ pixel scale. Each photometric image was obtained from 5 co-added dithered images, after sky subtraction and flat-field correction. The same photometric package as for the ST-7 data was used to perform photometry.

Table 2 (electronic form only) gives the results of our photometric monitoring.

\subsection{Optical spectroscopy}

The main source of spectroscopy is the $1.3 \mathrm{~m}$ Skinakas telescope $($ SKI), which was equipped with a $2000 \times 800$ ISA SITe CCD and a $13021 \mathrm{~mm}^{-1}$ grating, giving a nominal dispersion of $1.04 \AA /$ pixel. Many other spectra were obtained through the service programme of the Isaac Newton Group at La Palma, either with the $2.5 \mathrm{~m}$ Isaac Newton Telescope (INT) or the $4.2 \mathrm{~m}$ William Herschel Telescope (WHT). The INT was equipped with the Intermediate Dispersion Spectrograph (IDS), fit with the 235-mm camera and different intermediate-resolution gratings. The WHT spectra were obtained with the red arm of the Intermediate Dispersion Spectroscopic and Imaging System (ISIS), equipped with either the R600R or R1200R gratings.

Other observations have been obtained with the $2.6 \mathrm{~m}$ Nordic Optical Telescope (NOT), also located in La Palma, equipped with ALFOSC; the $1.93 \mathrm{~m}$ of the Haute Provence observatory (OHP) in France, equipped with the Carélec spectrograph and the Mt. Ekar $1.82 \mathrm{~m}$ Telescope (EKA) of the Padova Astronomical Observatory (Italy), equipped with AFOSC. One low resolution spectrum was taken with the blue arm of the TWIN spectrograph on the $3.5 \mathrm{~m}$ of the Calar Alto observatory (CA) in Spain. Table 3 (electronic form only) gives the log of the optical spectroscopic observations.

The reduction of the spectra was made using the STARLINK Figaro package (Shortridge et al. 2001), while their analysis was 
performed using the STARLINK Dipso package (Howarth et al. 1998).

\section{Results}

We have been monitoring 4U 0115+63 spectroscopically since the early 1990's (Paper II). Photometric data were also occasionally acquired. Since August 1999 the source is being monitored in the $U B V R I J H K$ bands. In this section we present the results of these new observations. The analysis of the historical variability curves will be presented in Sect. 4 .

\subsection{Photometric variability}

Figure 1 shows the evolution of the optical and infrared magnitudes and colours for the period August 2000-June 2006. These observations define a complete cycle of variability. The light curve begins with the photometric bright state that led to the 2000 X-ray outburst. The magnitudes gradually decreased and the colours became bluer. A faint stable photometric state was reached at around MJD 52000 (April 2001). This state extended for more than two years up to MJD 52880 (August 2003). We will refer to this state as the extended photometric low state (ELS). The average $B V R I$ magnitudes during the ELS are $B=$ $16.93 \pm 0.05, V=15.50 \pm 0.03, R=14.56 \pm 0.03, I=13.55 \pm 0.04$, $J=12.61 \pm 0.03, H=12.19 \pm 0.03, K=11.86 \pm 0.05$. These values are in complete agreement with those given in Paper I for the previous faint state. Photometrically, the ELS is a quiet state with very low amplitude variations, as can be deduced from the small values of the standard deviation.

The end of the ELS is marked by the gradual brightening of the photometric bands in August 2003. The source enters a new activity state characterised by large amplitude variations in the form of optical eruptions, i.e., the optical/IR brightness alternates between maxima and ELS values. The amplitude of variability is larger at longer wavelengths (e.g. $V$ increased by $\sim 0.5 \mathrm{mag}$ and $K$ by $1.6 \mathrm{mag}$ in about 440 days). In coincidence with the first optical eruption of this state, a new X-ray outburst was observed (September 2004). Note also the constancy of the $(B-V)$ colour throughout the observations.

Baykal et al. (2005) reported ROTSE observations covering the 2004 outburst. They found a sharp drop lasting for about a week (MJD 53235-53242) of $\sim 0.3 \mathrm{mag}$ in the source brightness a few days before the onset of the X-ray outburst and interpreted this result as a sign of mass ejection from the outer parts of the disc of the Be star. We do not observe such drop in our data. Although our observations contain only one point in the narrow interval when the ROTSE drop was seen, it shows very similar values to those of the previous and following observations.

\subsection{Spectroscopic variability}

Figure 1 also shows the evolution of the equivalent width $(\mathrm{EW}(\mathrm{H} \alpha))$ and the separation of the peaks, in $\mathrm{km} \mathrm{s}^{-1}$, of the split profiles of the $\mathrm{H} \alpha$ line in the interval 1999-2006. As the $\mathrm{EW}(\mathrm{H} \alpha)$ increases the peak separation decreases. This is the expected behaviour of a quasi-Keplerian disk and indicates that as the $\mathrm{EW}(\mathrm{H} \alpha)$ increases, the region where the $\mathrm{H} \alpha$ line is produced moves further away from the central star (e.g. Hummel \& Vrancken 1995).

In Fig. 2 the $\mathrm{H} \alpha$ profile through the different phases of the variability cycle is displayed. The top panel corresponds to the cycle that began after the 1995 outburst (Paper II), while
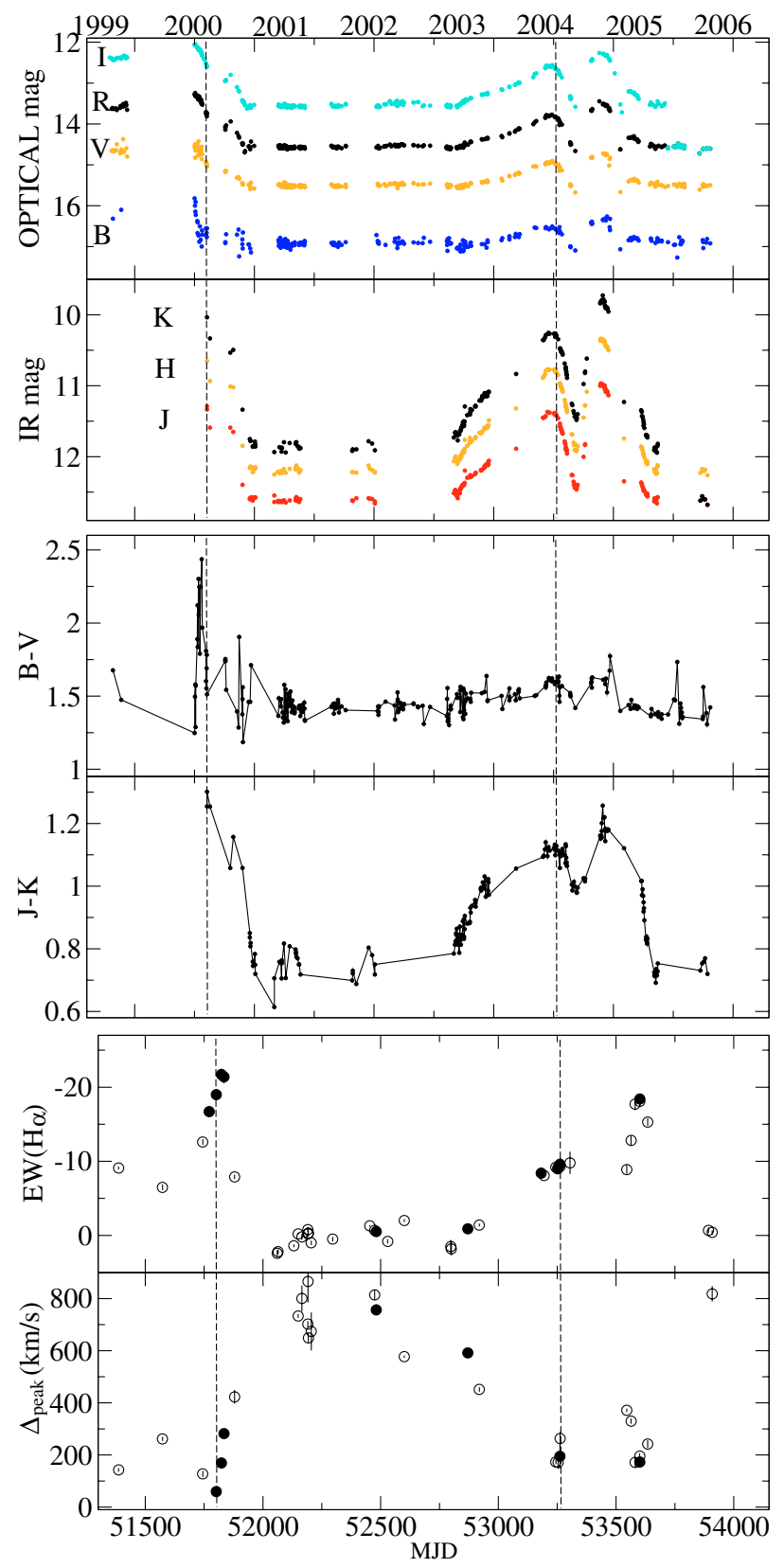

Fig. 1. Long-term evolution of the optical and infrared magnitudes and colours and of the $\mathrm{H} \alpha$ equivalent width and peak separation of doublepeak profiles. Dotted lines mark the occurrence of X-ray outbursts. Filled circles in the bottom panels correspond to the observations shown in Fig. 2.

the bottom panel to that after the 2000 outburst. The emission line profile of the $\mathrm{H} \alpha$ line shows significant richness in variability. Both single- and double-peak as well as symmetric and asymmetric profiles are seen. Symmetric profiles are associated with extreme values, either way, of the $\operatorname{EW}(\mathrm{H} \alpha)$. Asymmetric profiles are associated with intermediate values of $\operatorname{EW}(\mathrm{H} \alpha)$.

Taking as the starting point of the cycle the X-ray outburst prior to the ELS, the evolution of the $\mathrm{H} \alpha$ profile through the cycle is the following: at the time of the outburst the $\mathrm{H} \alpha$ line shows a single peak profile. The $\mathrm{EW}(\mathrm{H} \alpha)$ finds itself in a relative maximum. When the profile is a narrow single peak there is a broad base component on top of which it sits (see e.g. Fig. 2 spectra MJD 51771 and MJD 51801). This component was already noticed in Negueruela et al. (2001) and may be 


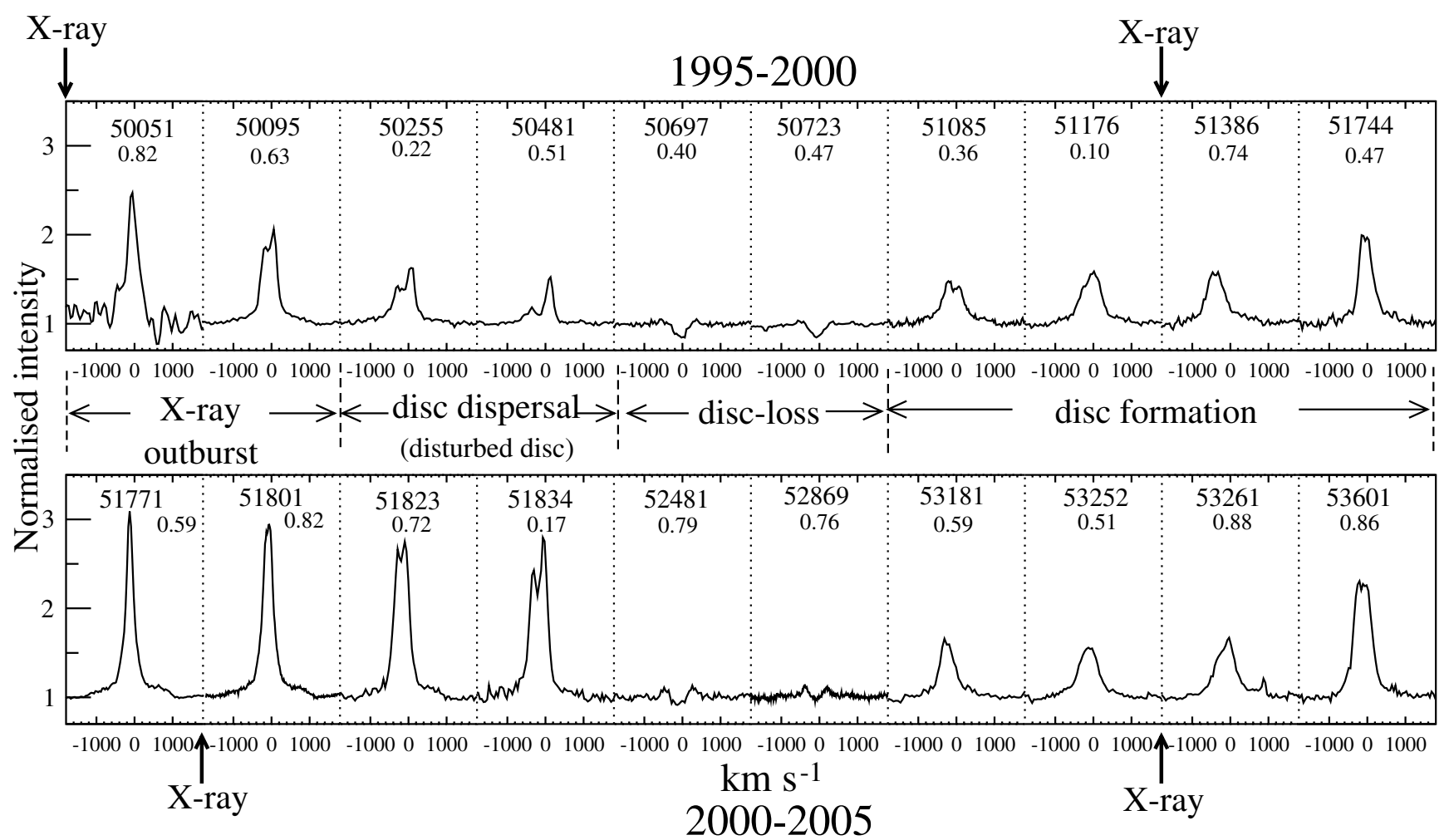

Fig. 2. Evolution of the $\mathrm{H} \alpha$ line profile over the $\sim 5$ year quasi-cycle. The spectra were normalised to the neighbouring continuum and the wavelength converted to velocity units. Indicated are the MJD and orbital phase according to the orbital solution of Tamura et al. (1992). The $Y$-axis scale was left the same in all panels to facilitate comparison.

interpreted as evidence for a warped disc. The broad base presumably represents the motion in the inner part of the disk, where the velocity components are higher. After the X-ray outburst the $\mathrm{H} \alpha$ profile becomes double peaked and asymmetric with the strength of the red peak larger than that of the blue peak ( $V<R$ phase). The $\mathrm{EW}(\mathrm{H} \alpha)$ decreases gradually until the source enters the ELS. At this point, $\mathrm{EW}(\mathrm{H} \alpha)$ presents its minimum value. Absorption as well as double-peak profiles are seen. For some spectra the depression between the double-peak profile extends below the stellar continuum. As the optical activity increases, the profile shows a distorted structure again, although not as marked as during the decay. At the beginning of this phase single-peak and red-dominated double-peak profiles are present. As the $\mathrm{EW}(\mathrm{H} \alpha)$ increases blue-dominated double-peak profiles start to appear. When the $\mathrm{EW}(\mathrm{H} \alpha)$ reaches $\sim 10 \AA$ a new X-ray outburst takes place. This outburst does not alter the strength of the $\mathrm{H} \alpha$ line significantly, although the overall brightness of the source decreases. By August 2005, the strength of the $\mathrm{H} \alpha$ line and the IR emission had experienced a fast and sudden increase. The $\operatorname{EW}(\mathrm{H} \alpha)$ almost doubled, while the IR magnitudes became $\sim 1$ mag brighter, on time scales of a few tens of days. It is as though the source was preparing itself for a new X-ray outburst. However, our latest observations (June 2006) show, both the $\mathrm{EW}(\mathrm{H} \alpha)$ and photometric magnitudes, close to ELS values and no evidence of renewed X-ray activity.

\subsection{Reddening and distance}

In Paper I values of the reddening and distance were derived from the observations showing the bluest colours (those from January 8, 1998). It was then assumed that these observations corresponded to purely photospheric emission. In view of the observations presented here, a revision of these parameters is justified since $i$ ) a much longer set of optical photometric observations is available, ii) a longer extended low optical state (MJD 52 000-52 800) is observed, where presumably the underlying B star is exposed and photospheric emission without substantial contribution from the disc is detected and iii) the observations cover a wider wavelength band with (quasi)simultaneous optical and near IR data, which allows us to estimate the reddening by fitting the photometric data to a model atmosphere. Figure 3 shows the energy distribution of a B0V star (Straizys 1995) and the lowest magnitudes of the ELS. Letting $A_{\mathrm{V}}$ be a free parameter we find that the best fit is achieved for $A_{\mathrm{V}}=$ $5.17 \pm 0.03$. The distributions with $A_{\mathrm{V}}= \pm 3 \sigma$ are also shown.

Taking into account that $A_{K}=0.115 A_{\mathrm{V}}$, that for a B0V the intrinsic $K_{0}=-3.25$ (Straizys 1995) and the minimum observed magnitude, $K=11.89 \pm 0.02$, the distance-modulus is $D M=$ $K-0.115 A_{\mathrm{V}}-K_{0}=14.55$ or $d=8.1 \pm 0.1 \mathrm{kpc}$. The error in the distance includes the photometric uncertainty only and assumes that neither $K_{0}$ nor the reddening law are affected by errors for example, an uncertainty of $0.2 \mathrm{mag}$ in $K_{0}$ translates into a distance error of about $0.7 \mathrm{kpc}$.

The assumption of standard reddening may introduce large errors if not verified. In Paper I, it was shown that the use of the extinction law by Fitzpatrick (1999) favours a value of $R$ close to the standard $R=3.1$. For further verification, we used the CHORIZOs code (Maíz-Apellániz 2004) to study the reddening. The program was used to fit extinction laws from Cardelli et al. (1989) convoluted with the spectral energy distribution of a $T_{\text {eff }}=27,500 \mathrm{~K}, \log g=4.0$ TLUSTY atmosphere model to our photometry. Although we obtain a slightly lower value for $R$, the derived $A_{\mathrm{V}}=4.8 \pm 0.1$ is compatible with the value found above at a $3 \sigma$ level. 


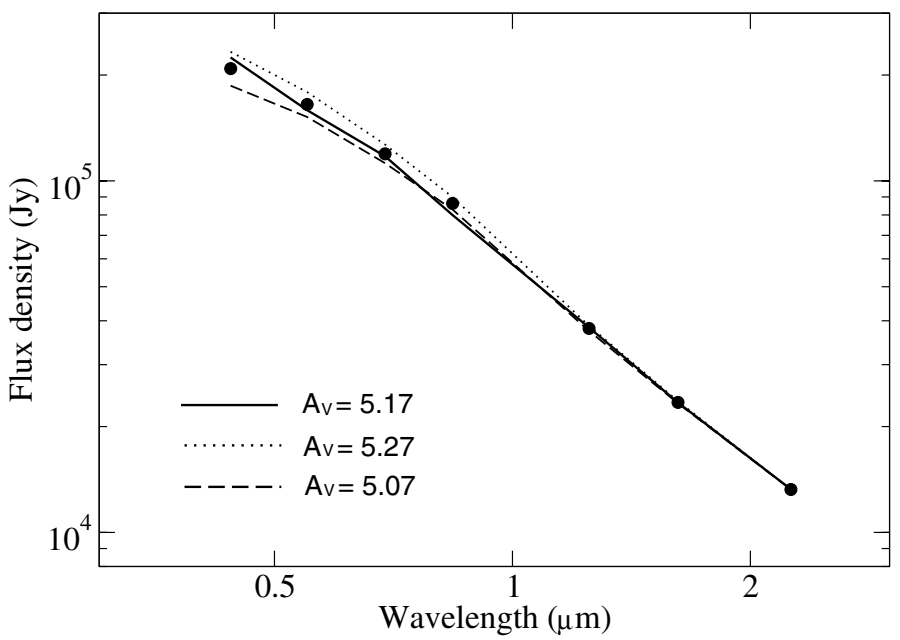

Fig. 3. Energy distribution of a B0V star dereddened using $A_{\mathrm{V}}=5.17$.

\subsection{Power spectrum analysis}

In order to search for periodic variations in the photometric light curves we performed a power spectral analysis. The details of the technique employed can be found in Larionov et al. (2001).

The power spectrum and spectral window of the data set were calculated as

$P(f)=\frac{1}{N^{2}}\left|\sum_{j=1}^{N}\left(m_{j}-\bar{m}\right) \mathrm{e}^{-\mathrm{i} 2 \pi f t_{j}}\right|^{2}$

and

$W(f)=\frac{1}{N^{2}}\left|\sum_{j=1}^{N} \mathrm{e}^{-\mathrm{i} 2 \pi f t_{j}}\right|^{2}, \quad W(0)=1$,

respectively, where $i=\sqrt{-1}, t_{j}$ denotes the time of observation, and $m_{j}$ and $\bar{m}$, the individual and mean values of the brightness, respectively.

It is clear from Fig. 1 that in order to judge with sufficient confidence the reality of the orbital plus close and/or related periodicities, one should remove the longer-period component(s) of variability. In our case it is not sufficient to subtract the linear trend, and we need to take into account the slow light variations. We have constructed a smoothed data set using the method of a sliding mean with the window value $\Delta$, replacing raw data $m_{i}$ for each time $t_{i}$ by the weighted mean:

$m_{i}^{\prime}=-2.5 \log \left(\frac{1}{\sum p_{j}} \sum_{j=1}^{k} p_{j} \cdot 10^{-0.4 m_{j}}\right)$,

where $k$ is the number of data points within interval $\left[t_{i}-\Delta, t_{i}+\Delta\right]$, and the weight of $j$ th point is determined as

$p_{j}=\exp \left[-\left(\delta t_{j} / \Delta\right)^{2}\right]$,

where $\delta t_{j}$ is the time span from the $j$ th point to the center of the window. The optimal value of the smoothing interval was searched by trial and error within a range $10^{\mathrm{d}}<\Delta<30^{\mathrm{d}}$. The criterion for $\Delta$ selection was the signal-to-noise ratio of the power-spectrum peaks in the region of interest, i.e. around the orbital frequency.

We divided the photometric light curves into several intervals and analysed them separately: MJD 51 400-52000 corresponds to the brighter optical state of the source prior to the
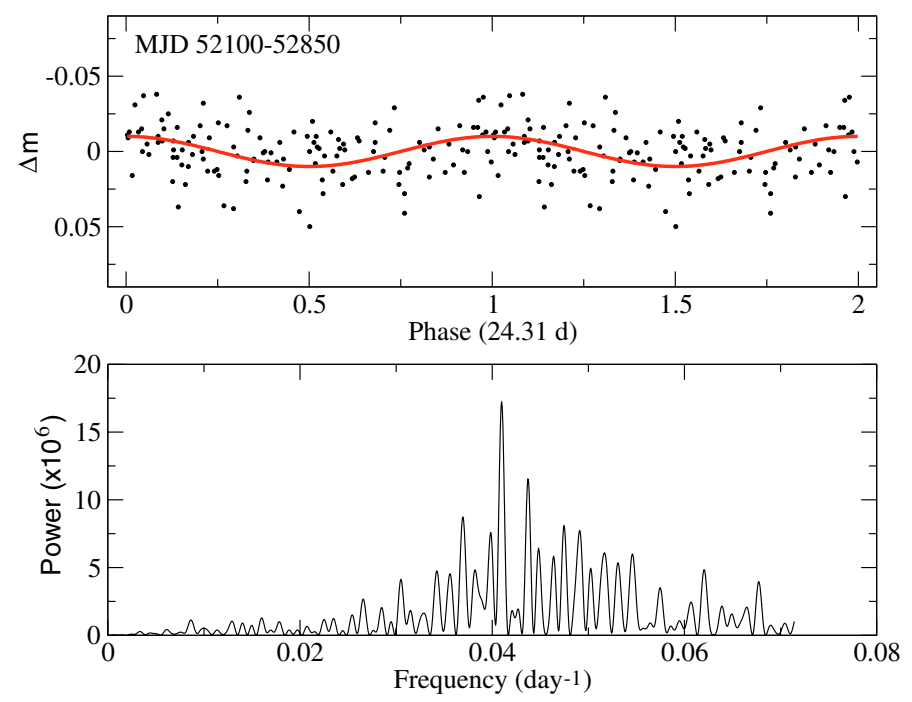

Fig. 4. Top: $R-R^{\prime}$ dependence on the phase of the 24.31-day period for the ELS. Bottom: power spectrum of the residuals $R-R^{\prime}$ obtained from the period MJD 52 100-52 300. The most prominent peak corresponds to $P=24.41$ days.

ELS, MJD 52 100-52850 corresponds to the ELS, MJD $52850-$ 53250 includes the smooth brightening after the ELS up to the 2004 X-ray outburst and MJD 53 250-53 715 covers the time interval after the X-ray outburst.

A clear modulation was found during the ELS, independent of the value of the smoothing interval adopted. The most prominent peak in the power spectrum corresponds to a period of $24.4 \pm 0.1 \mathrm{~d}$, which is consistent with the orbital period. The low-frequency components are most effectively suppressed and the highest signal-to-noise ratio is obtained when $\Delta=15^{\mathrm{d}}$. The power spectrum for the residuals $R_{j}-R_{j}^{\prime}$ is shown in Fig. 4.

The probability of chance occurrence of a peak with amplitude $P_{\max }$ in the power spectrum with a mean value $P_{\text {mean }}$ can be estimated as $\Phi=100 \% \cdot\left\{1-\left[1-\exp \left[-\left(P_{\max } / P_{\text {mean }}\right)\right]\right]^{N_{\text {ind }}}\right\}$; for non-uniformly spaced data the number of independent frequencies $N_{\text {ind }}=-6.362+1.193 \cdot N+0.00098 \cdot N^{2}$, where $N$ is the number of observations (Horne \& Baliunas 1986). In our case $N=301$ and $N_{\text {ind }}=389$; after subtraction of the slow component as described above (Eqs. (3) and (4)), $\Phi<0.2 \%$.

The amplitude of the sine-wave obtained is $0.01 \mathrm{mag}$ in $R$. We calculate the ephemeris of the small-scale optical variations as $\mathrm{JD}_{\text {Max }}=2449498( \pm 1.0)+24.41( \pm 0.15) \cdot E$, where $E$ is the epoch number. The most striking (but not unexpected) result is that these values practically coincide, within errors quoted, with the X-ray ephemeris. The position of the optical maximum corresponds to the periastron passage. The orbital period modulation is also present in the time interval MJD 52 850-53250, but the shape is markedly non-sinusoidal.

No evidence of a 24-day peak was found in the interval MJD 51400-52000. Instead, there is a modulation with 22.15 day period and amplitude $\approx 0.03$. One possible explanation for the 22.15-d period is that it represents the beat frequency between the orbital frequency and precession frequency of the disc. In this case the disc would precess with retrograde motion with a period of about 250 days. After the 2004 X-ray outburst all periodicities are suppressed.

We also applied the same analysis to the $V$ and $I$ bands. For the $I$ band we obtained practically the same results as in $R$, while in $V$ we were not able to detect any significant modulation close to the orbital period. The fact that the beat frequency is more 


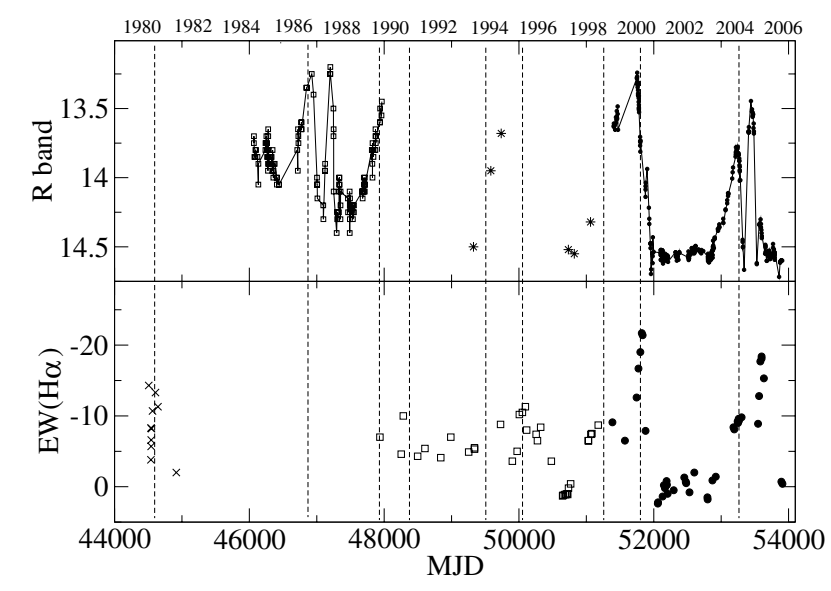

Fig. 5. Long-term evolution of the $R$ band and the $\operatorname{EW}(\mathrm{H} \alpha)$. In the top panel squares are from Mendelson \& Mazeh (1991) and stars from Negueruela et al. (2001). In the bottom panel squares are from Negueruela et al. (2001) and crosses from Kriss et al. (1983). The data from the year 2000 onward represent new observations. Dotted lines mark the occurrence of X-ray outbursts.

apparent in the redder bands gives support to the interpretation of the 22.15-d periodicity as being affected by the precession of the disc, as one would expect the IR magnitudes to be more affected by the disc that the shorter wavelengths.

\section{Discussion}

\subsection{Source states}

We have analysed optical light curves and spectra of 4U0115+63 spanning over more than two decades, the last 10 years with unprecedented coverage. During this period the source exhibited significant photometric and spectroscopic variability, allowing the definition of source states. The intensity of the optical, infrared and X-ray emission highly correlates with the state of the source.

Figure 5 shows the historical record (1980-2006) of the $R$ band and $\mathrm{H} \alpha$ equivalent width $(\mathrm{EW}(\mathrm{H} \alpha))$ of V635 Cas. Squares and stars in the top panel of Fig. 5 represent observations taken from Mendelson \& Mazeh (1991) and Negueruela et al. (2001), respectively. Squares in the $\mathrm{EW}(\mathrm{H} \alpha)$ panel are from Negueruela et al. (2001) and crosses from Kriss et al. (1983). Circles correspond to our new measurements. Vertical dashed lines denote the occurrence of X-ray outbursts. Note the striking repeatability of the pattern of variability during the periods 1986-1990 and 2002-2005: a photometric faint sate is followed by an optical flare with a slow gradual rise (200-300 days) and a fast decay (70-80 days). An X-ray outburst occurs when the source is in a photometric bright state. A second optical eruption separated from the previous one by about 250 days is not accompanied by X-ray activity. This second optical eruption presents a more symmetric profile with a fast rise and equally fast decay. Mendelson \& Mazeh (1991) also reported the presence of a small flare after the second eruption, peaking in June 1988 (MJD 47340) with an amplitude of $\sim 0.3$ mag and duration of 50 days. Exactly the same event is seen 17 years after (MJD 53 585). If the source follows the same pattern as in the late 1980's, then we should expect the source to remain in a low photometric state until spring 2007 and to start a new optical outburst soon after. The overall difference of $\sim 0.25$ mag between the Mendelson \& Mazeh's and our data set is most likely due to instrumental as well as calibration (selection of different secondary standard stars) effects, as no attempt to perform absolute photometry was made in Mendelson \& Mazeh (1991). The optical/IR/X-ray behaviour of 4U 0115+63/V635 Cas can be understood in terms of the evolution of the equatorial disc around the Be star. Two basic states can be distinguished depending on the presence or absence or the disc.

\subsubsection{The low state}

The low state would correspond to the complete loss of the disc or to a highly debilitated disc. During the low state, the source shows the weakest magnitudes, the bluest colours and the smallest $\mathrm{H} \alpha$ equivalent widths $(\mathrm{EW}(\mathrm{H} \alpha))$. The source exhibits little variability, with changes in the photometric bands of less than 0.05 magnitudes. Orbital modulation is detected. Since the source can stay in the low state for extended periods (a few years), the term "extended low state" (ELS) is used (Roche et al. 1993). During the low state the source is X-ray quiet. A discless state occurs when the $\mathrm{H} \alpha$ line displays an absorption profile. According to our interpretation of the long-term light curve shown in Fig. 6, 4U 0115+63 would have gone through ELS during MJD 48 600-49300 (1991-1993), MJD 50300-50900 (1996-1998) and MJD 52 000-52 800 (2001-2003).

The issue of whether the disc completely vanishes during the low state or there still exists some residual emission from a highly debilitated disc is a very important one, since in the case of total loss one can decouple the disc emission from that of the central star and hence determine the astrophysical parameters of the underlying star without any interference from the disc (Paper I). Disc-less states in 4U 0115+63 occurred in 1997 and 2001 as the $\mathrm{H} \alpha$ line appeared in absorption. Further support for the complete disappearance of the disc in 2001 comes from the fact that the standard deviation of the photometric magnitudes during this state does not follow any trend as a function of wavelength (see Sect. 3.1). Disc activity is expected to affect the redder magnitudes more than bluer magnitudes, hence making the IR magnitudes appear more variable. Also, from the power spectral analysis we observed that when the disc is present a beat period between the orbital period and presumably the precession period of the disc was found. Then the fact that no such beat frequency is detected during the ELS but only a lowamplitude ( $\sim 0.01 \mathrm{mag})$ modulation coinciding with the orbital period seems to indicate the absence of a disc. The 1992 low state would have not been accompanied by the complete loss of the disc as no absorption profile was attained.

The neutron star spins down during the low state. Previous studies have reported spin-up episodes throughout the duration of the X-ray outbursts (see e.g. Tamura et al. 1992). However, the historical record of the spin period hardly shows evidence for variability (see Table 1). Thus, if the neutron star spins up during the outburst, it must spin down in quiescence.

Disc-less phases have been seen in a number of Be/X-ray binaries but only two have been observed frequently enough as to allow a meaningful comparison with 4U 0115+63, namely, A $0535+26$ (Lyuty \& Zaitseva 2000; Larionov et al. 2001; Clark et al. 1999; Haigh et al. 2004) and X Per (Clark et al. 2001, and references therein). The three systems show extended low states, although its duration differs: 2600 days (MJD 50 700-51300) for A 0535+26 (Haigh et al. 2004; Zaitseva 2005), 800 days (MJD 52 000-52 800) for 4U 0115+63 (Fig. 1) and 1300 days (MJD 47 700-49000) for X Per (Clark et al. 2001). The longterm photometric light curve of X Per is very similar to that of 4U0115+63 (cf. Fig. 7 in Clark et al. 2001 with Fig. 1), 


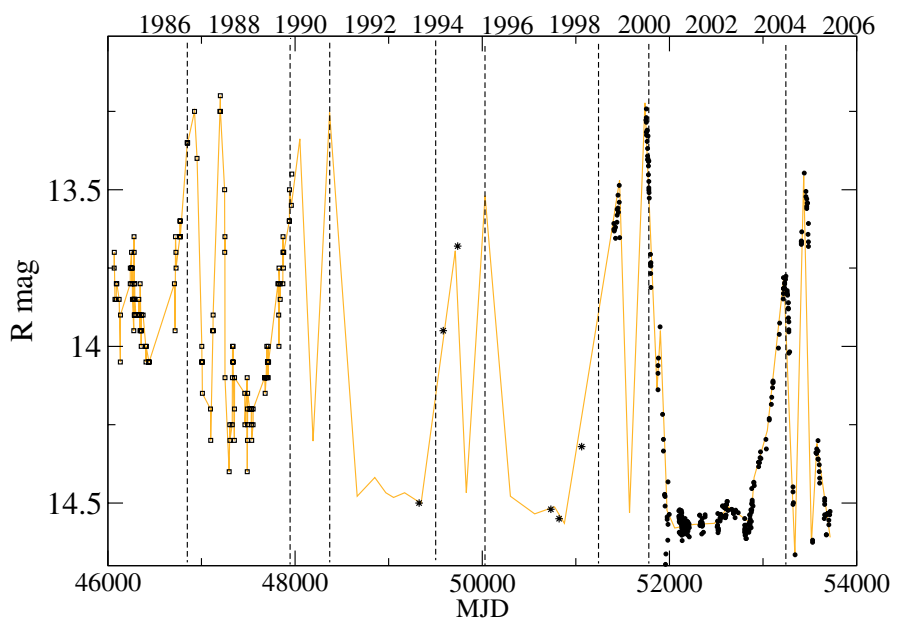

Fig. 6. The gray line represents our interpretation of the long-term light curve of V635 Cas, while the marked points correspond to real observations. Vertical dotted lines indicate the occurrence of X-ray outbursts. Low states occur in between pairs of outbursts, high states after the first outburst of the pair.

whereas A $0535+26$ exhibits a more choppy behaviour (see Fig. 1 in Clark et al. 1999).

\subsubsection{The high state}

As the disc reforms, it first behaves like the disc of an isolated Be star, growing in size. This phase is relatively smooth and it is characterised by a gradual increase in brightness and $\mathrm{EW}(\mathrm{H} \alpha)$. After the disc has reached its maximum size, further mass loss will result in an increase of the disc density, which can even become optically thick at IR wavelengths. At this point, the contribution of the disc to the optical/IR photometric magnitudes and colours is substantial. Fast ( $\sim 100$ days) and large amplitude variations (upto $1.5 \mathrm{mag}$ ) are seen. The source brightness alternates between low-state values and optical maxima. In this state the shape of the $\mathrm{H} \alpha$ line alternates between single-peak and doublepeak profiles, indicative of a perturbed disc.

X-ray outbursts always occur during the high state. They are preceded by the brightening of the optical and infrared emission. The $\mathrm{EW}(\mathrm{H} \alpha)$ reaches a relative maximum at the time of the X-ray peak. There seems to exist a triggering value of the $\mathrm{EW}(\mathrm{H} \alpha)$, around $10 \AA$, for the onset of the X-ray outburst. The $\mathrm{EW}(\mathrm{H} \alpha)$ prior to the start of the second outburst is always larger than that of the first one. Each X-ray outburst is then followed by a decrease in the optical/IR brightness but not always by the loss of the disc. Note that the $\mathrm{EW}(\mathrm{H} \alpha)$ remained fairly unaltered after the 1994, 1998 and 2004 X-ray outbursts (Fig. 5).

When the X-ray outburst does not lead to a low state (i.e. disc loss) the $\mathrm{EW}(\mathrm{H} \alpha)$ presents a relative minimum a few months after the outburst (Table 3 electronic form only, see also Table 1 in Paper II), as if the disc would have started a dispersal phase that never materialises. If this disc-loss phase does not take place, then another X-ray outburst occurs about 1-1.5 years later and is promptly followed by the complete disappearance of the disc. Indeed, a very interesting feature of the long-term light curve of $4 \mathrm{U} 0115+63$ is the fact that, for the last two decades, X-ray outbursts appear to have occurred in pairs, with two major outbursts happening in every cycle of disc build-up and loss (Fig. 5).

Although a comparison of the strength of the outbursts is hampered by the different energy ranges covered by the instruments that detected the events, we observe that the X-ray luminosity of the first outburst tends to be larger than the second one (Table 1). In one occasion (in 1996, MJD 50300-50400) the second X-ray outburst was followed by a series of orbital modulated minor outburst (so-called type I) (Negueruela et al. 1998).

The second outburst of the pair, however, is not always detected. This seems to be the case in the last cycle (2000-2005). Photometric magnitudes are back to low state values and 1.5 years have passed since the September 2004 outburst. Our latest spectra taken on June 2006 give values of the $\operatorname{EW}(\mathrm{H} \alpha)$ of $\sim-0.4 \AA$ and an absorption profile with two weak emission shoulders, a feature typically seen in small disks. This, however, does not necesarily mean that 4U0115+63 has returned to the low state. In Paper II we showed that fast transitions between single-peak and shell profiles are indicative of a precessing warped disc. Another missing outburst occurred during the 1985-1990 cycle (Mendelson \& Mazeh 1991). The similarity between the 2003-2006 and 1986-1989 light curves is striking. The second optical eruption in 1988 was not accompanied by an X-ray outburst either. Therefore we suggest that the intervals MJD 46700-48600 (1986-1991) and MJD 52 800-present (2003-present) should be considered as extended high states. Extended high states would occur in association with missing X-ray outbursts. Normal high states would last for about 3 years - the intervals MJD 47 600-48 600 (19891991), MJD 49300-50300 (1994-1996) and MJD 5090052000 (1998-2000) would correspond to high states - while extended ones would cover the entire cycle of 5 years. In this case no disc-less phase is achieved.

\subsection{The viscous decretion disc model}

The long-term variability of $4 \mathrm{U} 0115+63$ can be summarised as follows: the equatorial disc around the Be star dictates the state of the source. The quasi-cyclic variations coincide with the time scales for disc build-up and loss. X-ray outbursts occur in pairs, although the second one may be missing. In between pairs of outbursts the optical/IR emission and variability is highly suppressed, while in between the outbursts of a given pair the optical/IR emission is highly variable.

This behaviour can be explained within the framework of the truncated decretion disc model (Paper I, Okazaki \& Negueruela 2001; Okazaki et al. 2002). Initially, the disc grows gradually like in isolated Be stars. However, in Be/X-ray binaries the surface density of the disc increases more rapidly that that of isolated Be stars, as a consequence of truncation. The truncation of the disc is the result of the resonant torque exerted by the neutron star, which removes angular momentum from the disc. Okazaki et al. (2002) locates the truncation radius of $4 \mathrm{U} 0115+63$ at $0.36-0.39 a$, where $a$ is the semi-major axis of the orbit, for a wide range of values of the viscosity. The disc becomes optically thick at IR wavelengths and unstable to radiation-driven warping. Eventually, the disc begins to warp, tilt and precess.

The photometric variations observed during the high states (1987-1989, 2003-2005), characterised by large ( $\gtrsim 1 \mathrm{mag})$ and relatively fast ( $\sim 100$ days) amplitude variations, are rather large when compared to typical values seen in isolated Be stars (Dachs et al. 1988). In addition, the brightest magnitudes are accompanied by historic maxima of the $\operatorname{EW}(\mathrm{H} \alpha)$. These extreme values of the $\operatorname{EW}(\mathrm{H} \alpha)$ correspond to line shapes much narrower than at other times. In the framework of the disc deccretion model, the coincidence of very high values of the $\operatorname{EW}(\mathrm{H} \alpha)$ with narrow, single-peaked lines and very bright optical and infrared 
magnitudes during the high state, leads to the conclusion that extreme values happen because the disk becomes warped and occasionally presents a much higher surface to the observer (see Paper II). Therefore the changes in magnitudes should be the consequence of two different effects: on the one hand, an increase in the disk size or density; on the other hand, a change in the emitting surface observed. They should not be interpreted as fast phases of disc loss and reformation, despite the fact that the photometric magnitudes fall to ELS values. This strongly disturbed phase would begin after the first outburst.

One possible explanation for the difference between the first and second outburst could be the different state of the disc, with the first one occurring when the disc is still in a quasi-stable state while the second one when the disc has been distorted. The distortion of the disc leads to the interaction with the orbiting neutron star. If the interpretation of the 22.15 day period (Sect. 3.4) as the beat period between the orbital period and precession is correct, then the time scales associated with the perturbed disc $(\sim 250 \mathrm{~d})$ would be a factor $\sim 2$ longer than the duration of the optical eruption associated with the missing X-ray outburst $(\sim 150 \mathrm{~d})$. In this context the missing outburst would simply reflect the fact that no interaction between the neutron star and the distorted disc took place during the $\sim 6$ orbits that the optical eruption lasted.

An alternative explanation for the missing X-ray outburst requires the formation of an accretion disc around the neutron star (Mendelson \& Mazeh 1991) and it is based on the centrifugal inhibition at the edge of the neutron star magnetosphere (Stella et al. 1986). In the 1988 and 2005 events the matter accumulated in the disc would not be enough to overcome the propeller effect.

The $(B-V)$ colour index appears as a good indicator to distinguish between the two outbursts. While the $(J-K)$ colour (also $(V-R)$ and $(R-I)$, not shown in Fig 1$)$ follows smoothly the state of the disc, $(B-V)$ is rather insensitive to photometric or spectral changes. Only prior to the destructive outburst of September 2000 does it display high amplitude variability (Fig. 1).

In addition to the missing outbursts, the fact that the first X-ray outburst does not modify, at least dramatically, the strength of the $\mathrm{H} \alpha$ line also needs to be explained. In this respect, one may wonder how much of a disruption an X-ray outburst represents. As an order of magnitude estimate, we can derive the mass content in the disc simply as

$M=\int \rho \mathrm{d} V$

with $\rho=\rho_{0}\left(R_{*} / r\right)^{n}$ and $\mathrm{d} V=2 \pi r H \mathrm{~d} r$. Here $\rho_{0}$ is the density and the inner radius of the disc (at $r \sim R_{*}$ ), $R_{*}$ the star radius, $H$ the disc height and $n$ an exponent defining the density law. According to Waters et al. (1988) $n$ varies in the range $2-4$ in most BeX. The typical radius of a B0.2 star, is $R_{*}=8 R_{\odot}$ and assuming typical values for the disc radius $R_{\mathrm{d}}=5 R_{*}$, and inner density $\rho_{0}=10^{-10} \mathrm{~g}$ (Telting et al. 1998) $\mathrm{cm}^{-3}$ and $H=0.03 R_{*}$ (Negueruela \& Okazaki 2001) the mass is estimated to be $2.6 \times$ $10^{-9} M_{\odot}$ for $n=2$ and $7.8 \times 10^{-10} M_{\odot}$ for $n=4$.

On the other hand, a mean X-ray luminosity of $10^{37} \mathrm{erg} \mathrm{s}^{-1}$ for about a month requires the transfer of $\dot{M}=L_{x}(R / G M)=$ $5.4 \times 10^{16} \mathrm{~g} \mathrm{~s}^{-1}$ or $7 \times 10^{-11} M_{\odot}$ month $^{-1}$, that is just $\lesssim 10 \%$ of the mass in the disc. This relatively small effect on the mass content of the disc would explain the fact that the strength of the $\mathrm{H} \alpha$ line does not change much after the first outburst (see also Norton et al. 1994).

\section{Conclusion}

We have presented the results of our monitoring of the Be/X-ray binary 4U 0115+63/V635 Cas. Our 2000-2006 data represent the most complete optical and infrared photometric study of 4U 0115+63/V635 Cas made up to now. The combination of our observations over the last decade with published data allowed us to investigate the correlation between the optical, infrared and X-ray emission. X-ray outbursts, which come into pairs, occur when the source is photometrically bright and the $\mathrm{H} \alpha$ line appears strongly in emission. The equatorial disc around the $\mathrm{Be}$ star dictates the state of the source: if the disc is absent the source is in the low state. In the high state large amplitude variation and asymmetric spectral lines denotes a disturbed disc. The time scale for loss and build-up of the equatorial disc is 5 years, approximately 3 of which correspond to the high state and 2 to the low state. However, this quasi-periodic behaviour is broken if one of the X-ray outburst is missing. In this situation the high state covers the entire cycle. Bright magnitudes are observed because the disc warps and presents a larger surface to the observer. The fact that the infrared and optical magnitudes and the $\mathrm{H} \alpha$ equivalent width do not change dramatically after the first outburst may indicate that, at the time of the outburst, the disc had not been distorted yet. It is after the first outburst that the disc becomes unstable, warps and tilts. The second X-ray outburst takes place during this phase of strongly disturbed disc. The disc finally disappears, partly reabsorbed by the B star and partly used up to power the X-ray outburst.

Acknowledgements. We thank A. Manousakis, A. Di Paola and M. Dolci for their help in some of the observations. IN is a researcher of the programme Ramón y Cajal, funded by the Spanish Ministerio de Educación y Ciencia (MEC) and the University of Alicante, with partial support from the Generalitat Valenciana and the European Regional Development Fund (ERDF/FEDER). This research is partially supported by the MEC under grant AYA2005-00095. VL and AA acknowledge hospitality of the Rome and Teramo observatories during the observations in Campo Imperatore. This work has greatly benefited from the ING service programme. Skinakas Observatory is a collaborative project of the University of Crete, the Foundation for Research and Technology-Hellas and the Max-Planck-Institut für Extraterrestrische Physik. The near-infrared data were obtained with AZT-24 telescope operated under agreement between Pulkovo, Rome and Teramo observatories. The INT and JKT are operated on the island of La Palma by the Isaac Newton Group in the Spanish Observatorio del Roque de Los Muchachos of the Instituto de Astrofísica de Canarias. This research is partially based on data from the ING Archive. Part of the data presented here have been taken using ALFOSC, which is owned by the Instituto de Astrofísica de Andalucía (IAA) and operated at the Nordic Optical Telescope under agreement between IAA and the NBIfAFG of the Astronomical Observatory of Copenhagen. Partially based on observations obtained at the Observatoire de Haute Provence (CNRS, France), observations obtained at the Asiago observatory and the Centro Astronómico Hispano Alemán (CAHA) at Calar Alto, operated jointly by the Max-Planck-Institut für Astronomie and the Instituto de Astrofísica de Andalucía (CSIC). This research has made use of NASA's Astrophysics Data System Bibliographic Services and of the SIMBAD database, operated at the CDS, Strasbourg, France.

\section{References}

Baykal, A., Kiziloglu, U., Kiziloglu, N., Balman, S., \& Inam, S. C. 2005, A\&A, 439, 1131

Cardelli, J. A., Clayton, G. C., \& Mathis, J. S. 1989, ApJ, 345, 245

Clark, J. S., Lyuty, V. M., Zaitseva, G. V., et al. 1999, MNRAS, 302, 167

Clark, J. S., Tarasov, A. E., Okazaki, A. T., Roche, P., \& Lyuty, V. M. 2001, A\&A, 380, 615

Coe, M. J. 2000, ASPC, 214, 656

Cominsky, L., Clark, G. W., Li, F., Mayer, W., \& Rappaport, S. 1978, Nature, 273,367

Cominsky, L., Roberts, M., \& Finger, M. H. 1994, in The Second Compton Symposium, AIP Conf. Proc. 304, ed. C. E. Fichtel, N. Gehrels, \& J. P. Norris (New York, NY: AIP Press), 294

Dachs, J., Engels, D., \& Kiehling, R. 1988, A\&A, 194, 167

Finger, M. H., Scott, M., Hagedon, K., et al. 1995, IAUC, 6266 
Fitzpatrick, E. L. 1999, PASP, 111, 63

Forman, W., Jones, C., \& Tananbaum, H. 1976, ApJ, 208, 849

Haigh, N. J., Coe, M. J., \& Fabregat, J. 2004, MNRAS, 350, 1457

Heindl, W. A., Coburn, W., Gruber, D. E., et al. 1999, ApJ, 521, L49

Horne, J. H., \& Baliunas, S. L. 1986, ApJ, 302, 757

Howarth, I. D., Murray, J., Mills, D., \& Berry, D. S. 1998, Starlink User Note, 50.21

Hummel, W., \& Vrancken, M. 1995, A\&A, 302, 751

Hutchings, J. B., \& Crampton, D. 1981, ApJ, 247, 222

Johns, M., Koski, A., Canizares, C., \& McClintock, J. 1978, IAUC, 3171

Kelley, R. L., Brodheim, M. J., Cominsky, L., Stothers, R., \& Rappaport, S. 1981, ApJ, 251, 630

Kriss, G. A., Cominsky, L. R., Remillard, R. A., Williams, G., \& Thorstensen, J. R. 1983, ApJ, 266, 806

Larionov, V., Lyuty, V. M., \& Zaitseva, G. V. 2001, A\&A, 378, 837

Lutovinov, A. A., Grebenev, S. A., \& Sunyaev, R. A. 2000, AstL, 26, 1

Lyuty, V. M., \& Zaitseva, G. V. 2000, AstL, 26, 9

Maíz-Apellániz, J. 2004, PASP, 116, 859

Mendelson, H., \& Mazeh, T. 1991, MNRAS, 250, 373

Negueruela, I., Reig, P., Coe, M. J., \& Fabregat, J. 1998, A\&A, 336, 251

Negueruela, I., \& Okazaki, A. T. 2001, A\&A, 369, 108 (Paper I)

Negueruela, I., Okazaki, A. T., Fabregat, J., et al. 2001, A\&A, 369, 117 (Paper II)

Norton, A. J., Chakrabarty, D., Coe, M. J., et al. 1994, MNRAS, 271, 981

Okazaki, A. T., \& Negueruela, I. 2001, A\&A, 377, 161

Okazaki, A. T., Bate, M. R., Olgivie, G. I., \& Pringle, J. E. 2002, MNRAS, 337, 967

Rappaport, S., Clark, G. W., Cominsky, L., Li, F., \& Joss, P. C. 1978, ApJ, 224, L1
Ricketts, M. J., Hall, R., Page, C. G., \& Pounds, K. A. 1981, SSRv, 30, 399

Roche, P., Coe, M. J., Fabregat, J., et al. 1993, A\&A, 270, 122

Rose, L. A., Marshall, F. E., Holt, S. S., et al. 1979, ApJ, 231, 919

Santangelo, A., Segreto, A., \& Giarrusso, S. 1999, ApJ, 523, L85

Sazonov, S., \& Sunyaev, R. 1995, IAUC, 6272

Scott, D. M., Finger, M. H., Wilson, R. B., Prince, T. A. 1994, IAUC, 5990

Scott, M., Finger, M. H., Wilson, R. B., Prince, T. A., Vaughan, B. 1996, IAUC, 6450

Shortridge, K., Meyerdierks, H., Currie, M., et al. 2001, Starlink User Note 86.19 Stella, L., White, N. E., \& Rosner, R. 1986, ApJ, 308, 669

Straizys, V. 1995, Multicolor stellar photometry, Astronomy and Astrophysics Series, Vol. 15 (Tucson, Arizona: Parchart Publishing House)

Tamura, K., Tsunemi, H., Kitamoto, S., Hayashida, K., \& Nagase, F. 1992, ApJ, 389,676

Telting, J. H., Waters, L. B. F. M., Roche, P., et al. 1998, A\&A, 339, 150

Tsunemi, K. \& Kitamoto, S. 1988, ApJ, 334, L21

Tueller, J. 2004, IAUC, 8411

Waters, L. B. F. M., van den Heuvel, E. P. J., Taylor, A. R., Habets, G. M. H. J., \& Persi, P. 1988, A\&A, 198, 200

Wheaton, W. A., Doty, J. P., Primini, F. A., et al. 1979, Nature, 282, 240

White, N. E., Swank, J. H., \& Holt, S. S. 1983, ApJ, 270, 711

Whitlock, L., Roussel-Dupre, D., \& Priedhorsky, W. 1989, ApJ, 338, 381

Wilson, R. B., Finger, M. H., \& Scott, D. M. 1994, IAUC, 5999

Wilson, R. B., Harmon, B. A., \& Finger, M. H. 1999, ATel, 40

Unger, S. J., Roche, P., Negueruela, I., et al. 1998, A\&A, 336, 960

Zaitseva, G. V. 2005, AstL, 31, 103

Ziolkowski, J. 2002, MmSAI, 73, 1038

Zurita Heras, J. A., Shaw, S. E., Kreykenbohm, I., et al. 2004, ATel, 331 